\title{
Terapia médica de la ginecomastia con tamoxifeno. Influencia del volumen y duración de la ginecomastia en el resultado terapéutico
}

\author{
Enzo Devoto $C^{1}$, Marcia Madariaga $A^{2 a}$, Ximena Lioi $C^{1}$, \\ Nelly Mardones ${ }^{3}$
}

\author{
Influence of size and duration \\ of gynecomastia on its response \\ to treatment with tamoxifen
}

\begin{abstract}
Background: Gynecomastia is treated when it is painful, there are psychosocial repercussions or it does not revert in less tan two years. It is treated with the antiestrogenic drug tamoxifen, but there are doubts about its effectiveness in high volume gynecomastias or in those lasting more than two years. Aim: To assess the effectiveness and safety of tamoxifen for gynecomastia and the influence of its volume and duration on the response to treatment. Patients and methods: Forty three patients with gynecomastia, aged 12 to 62 years, were studied. Twenty seven patients had a pubertal physiological gynecomastia, in eight it was caused by medications, in four it was secondary to hypogonadism, in three it was idiopathic and in one it was due to toxic exposure. Twenty patients had mastodynia and in 33, gynecomastia had a diameter over $4 \mathrm{~cm}$. It lasted less than two years in 30 patients, more than two years in nine and four did not recall its duration. All were treated with tamoxifen $20 \mathrm{mg} /$ day for 6 months. A follow up evaluation was performed at three and six months of treatment. Results: Mastodynia disappeared in all patients at three months. At six months gynecomastia disappeared in 26 patients (62\%), but relapsed in 27\%. All gynecomastias caused by drugs with antiandrogen activity disappeared. Fifty two percent of gynecomastias over $4 \mathrm{~cm}$ and $90 \%$ of those of less than $4 \mathrm{~cm}$ in diameter disappeared $(p<0.05)$. Fifty six percent of gynecomastias lasting more than two years and $70 \%$ of those of a shorter duration disappeared $(p=N S$ ). Two patients had diarrhea or flushes associated to the therapy. Conclusions: Tamoxifen is safe and effective for the treatment of gynecomastia. Larger lesions have a lower response to treatment (Rev Méd Chile 2007; 135: 1558-65).
\end{abstract}

(Key words: Androgen antagonists; Estrogen antagonists; Gynecomastia; Tamoxifen)

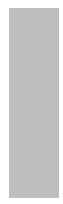

Recibido el 25 de septiembre, 2006. Aceptado el 17 de julio, 2007).

${ }^{1}$ Sección Endocrinología, Servicio de Medicina, Hospital Clínico San Borja Arriarán, Departamento de Medicina, Facultad de Medicina, Campus Centro, Universidad de Chile. ${ }^{2}$ Instituto de Investigaciones Materno Infantil, Facultad de Medicina, Campus Centro, Universidad de Chile. Santiago de Chile. ${ }^{3}$ Departamento de Endocrinología, Hospital Félix Bulnes.

aTecnólogo Médico.

Correspondencia a: Dr. Enzo Devoto C. Hernando de Aguirre 194 of 62. Fax: 2290238. Santiago, Chile.

E-mail: edevoto@vtr.net 
$\mathrm{L}^{2}$ a ginecomastia (Gi) es el aumento de tamaño de la glándula mamaria en el hombre. Es un signo clínico y no una enfermedad ${ }^{1}$, debe diagnosticarse si se trata de Gi patológica o fisiológica antes de realizar un tratamiento médico o quirúrgico ${ }^{2}$. La Gi patológica puede ser producida por fármacos o alteraciones en el balance estrógeno (E) - testosterona (T) debido a cuadros funcionales o tumorales ${ }^{1,2}$.

Aún se discute cómo y cuándo estudiar una ginecomastia. Nuestro grupo lo realiza siguiendo la clasificación de Gi y estudio de flujo descrito en anterior trabajo ${ }^{3}$.

De esta forma, si se logra terapéuticamente la desaparición de la ginecomastia sin investigar su causa, puede retardarse el diagnóstico etiológico y la posibilidad de una terapia específica exitosa, como por ejemplo, tumor adrenal feminizante ${ }^{4}$, tumor testicular ${ }^{5}$ y lesión orgánica hipotalamohipofisiaria $^{2}$. En Gi causada por síndrome de Klinefelter ${ }^{6} \mathrm{u}$ otra causa que requiera terapia de reemplazo androgénica, el postergar su inicio favorece el desarrollo de complicaciones, como disminución de masa ósea.

La mayoría de los pacientes portadores de Gi fisiológica no requieren tratamiento, ya que generalmente desaparece en un período de 2 años ${ }^{2,7}$; en la patológica de causa farmacológica habitualmente remite al discontinuar la droga.

Existen situaciones en que la Gi tanto fisiológica como patológica debe tratarse para hacer desaparecer el dolor o reducir el tamaño, lo que se logra por terapia médica o quirúrgica.

Ha predominado la extirpación quirúrgica del tejido mamario, no estando exenta de complicaciones y recidiva ${ }^{8}$.

Se plantea como alternativa la terapia médica con antiestrógenos e inhibidores de la aromatasa.

El estrógeno favorece el crecimiento mamario, su acción se inicia uniéndose a su receptor celular. El complejo estrógeno-receptor (E-R) actúa a nivel nuclear, desencadenando el proceso de proliferación celular'? El antiestrógeno se liga al E-R impidiendo su acción proliferativa mamaria; en este mecanismo se fundamenta su utilidad terapéutica en cáncer de mama ${ }^{10}$ y en Gi.

El primer antiestrógeno utilizado fue el citrato de clomifeno en dosis de 50 a $100 \mathrm{mg} /$ día, en estudios no controlados logra entre 40 y $90 \%$ de eficacia terapéutica ${ }^{11-13}$.
En 16 pacientes portadores de Gi puberal tratados con $50 \mathrm{mg} /$ diarios de clomifeno, nuestro grupo logró desaparición de la Gi en 62\% de los $\operatorname{casos}^{14}$

Desde la década 1980-89, el clomifeno fue reemplazado por el tamoxifeno en dosis de 10 a $20 \mathrm{mg} /$ día $^{15-18}$. Este fármaco, además de la acción antiestrógenica, disminuye los factores de crecimiento Transforming Growth Factor alfa (TGFa) y el Insulin Growth Factor 1 (IGF-1) implicados en la proliferación del tejido mamario?.

Las series publicadas con clomifeno y tamoxifeno de hasta 6 meses de tratamiento, no reportan efectos colaterales importantes; en cáncer de mama tratado con tamoxifeno se han comunicado complicaciones tromboembólicas ${ }^{10}$.

La histopatología de la Gi señala que en las primeras etapas hay proliferación del epitelio ductal, en el estroma, el componente es hiperplásico y congestivo edematoso; evoluciona con el transcurso del tiempo a un predominio de hialinización y fibrosis ${ }^{2}$. Basados en estos hechos morfológicos, se recomienda la terapia médica en las Gi de corta duración ${ }^{1,2}$ antes que se produzcan cambios tisulares que no regresen.

Realizamos un trabajo descriptivo, retrospectivo y no controlado de pacientes con Gi tratados con tamoxifeno en la sección de Endocrinología, de acuerdo a pautas pre-establecidas de diagnóstico y terapia.

Sus objetivos fueron evaluar el efecto del tamoxifeno en desaparición de mastalgia y en reducción o desaparición del aumento de volumen mamario.

Se analizó si el tamaño y la duración de la Gi influyen sobre el resultado terapéutico.

\section{Pacientes Y MÉTOdo}

Cuarenta y tres pacientes portadores de ginecomastia, de 12 a 62 años, de los que se obtuvo su consentimiento informado, cumplieron con uno o más de los criterios que adoptamos para tratar Gi:

Gi puberal que no ha regresado en 2 años o continúa en crecimiento $(n=9)$; mastalgia $(n=20)$; crecimiento rápido. La literatura no lo define, para nosotros es un crecimiento mamario de $4 \mathrm{~cm} \mathrm{o}$ más en $\leq 1$ año $(n=9)$. Cuando hay repercusión 
psicosocial y deterioro de la imagen $(n=10)$; en la inducida por fármacos que no regresa post suspensión o cuando es inconveniente suspender el fármaco $(n=7)$.

El período de 2 años en que debería haber regresado una Gi puberal, se eligió por datos de la literatura $^{1,7}$ y por resultados de un grupo control de 11 varones portadores de Gi puberal, edad promedio 12 años 10 meses, ( $r=10-14)$ con seguimiento de 22 meses, el promedio para alcanzar la regresión fue 15 meses $(r=6-22)$.

No se incluyeron pacientes con índice de masa corporal > 30 en el adulto y mayor al percentil 95 ajustado por edad y sexo, en el púber y adolescente, para facilitar la semiología mamaria dificultada por la obesidad.

La etiología de la Gi fue: fisiológica de la pubertad 27, (11\% Tanner II, 19\% Tanner III, 33\% Tanner IV, 37\% Tanner V), patológica 13 e idiopática del adulto 3.

No hubo Gi fisiológicas del adulto mayor, los pacientes de 60 y 62 años eran portadores de Gi patológica.

En Gi patológica: hipogonadismo 4 (hipogonadotropo 2 e hipergonadotropo 2), tóxico 1, farmacológica 8: cimetidina, ketoconazol y antiandrógenos (etinilestradiol-ciproterona, espironolactona y flutamida), estos últimos utilizados con éxito en acné resistente a la terapia dermatológica.

En el examen físico, la presencia de tejido mamario se diferenció del aumento de volumen por tejido adiposo o lipomastia, cogiendo el tejido entre pulgar e índice y comparándolo con igual maniobra realizada a nivel del pliegue axilar anterior; el tejido mamario es firme, muy diferente a la consistencia de la piel y grasa axilar ${ }^{19}$.

Para determinar el tamaño inicial de la Gi y su cambio bajo terapia, se desplazó el pulgar del examinador (E. D.) desde el pezón hacia la periferia en sentido vertical y transversal; se determinaron los márgenes externo e interno para el diámetro transversal y superior e inferior para el vertical. Con regla se midió la distancia entre el pulgar y el índice ubicados en dichos bordes estableciéndose el diámetro máximo del tejido mamario medido en centímetros.

Referente al aumento de tamaño mamario, en 33 pacientes era $\geq 4 \mathrm{~cm}$ de diámetro máximo, y en 10 pacientes $<4 \mathrm{~cm}$.
Respecto a la duración de la Gi, 39 de 43 pacientes aportaron esta información: 30 con una duración de 2 años o menos ( $r=1$ mes - 2 años) y 9 con más de 2 años ( $r=3-6$ años).

De los 43 pacientes tratados con tamoxifeno, 12 tenían $>20$ años de edad $(r=21-62)$ y $31<20$ años ( $r=12-19)$. En Gi patológica, de ser posible se trató la causa específica y se realizó la terapia antiestrogénica de cumplirse los criterios antes señalados. Si la Gi era producida por fármaco y no era conveniente suspenderlo, como en tratamiento exitoso de acné severo con antiandrógeno, este se mantuvo y se agregó el antiestrógeno.

Los pacientes fueron tratados con $20 \mathrm{mg}$. diarios de tamoxifeno en dosis única, por 6 meses.

Los controles se realizaron a los 3 y 6 meses de terapia, evaluándose la respuesta por desaparición del dolor y variación del tamaño, el que se objetivó por cambios en el diámetro máximo. Se determinó el porcentaje de disminución del tamaño o la desaparición completa de la $\mathrm{Gi}$; se interrogó sobre síntomas colaterales.

De acuerdo a esta información se clasificó la respuesta terapéutica como:

Buena: sin mastalgia y sin ginecomastia. Regular: sin mastalgia y reducción de $\geq 50 \%$ del diámetro máximo. Mala: dolor sin variación o reducción de $<50 \%$ del diámetro máximo o referencia a cirugía

Los pacientes con desaparición de Gi se controlaron hasta el año post-suspensión del tratamiento para evaluar recidiva. No se contó con grupo de control durante la terapia, ni estudio ecográfico mamario.

En $2 / 3$ de los pacientes con Gi puberal, se inició terapia antes de 2 años de su aparición.

Se evaluó la influencia en el resultado terapéutico del tamaño de la Gi $(\geq$ de $4 \mathrm{~cm}$. y $<$ de $4 \mathrm{~cm}$ ) y su duración ( $\leq 2$ años y $>2$ años)

Análisis estadístico: Chi-cuadrado y Fisher exact test.

\section{Resultados}

De 43 pacientes con Gi, 20 presentaban mastalgia, a los 3 meses de tratamiento el dolor desapareció en $100 \%$, generalmente entre el primer y segundo mes. 


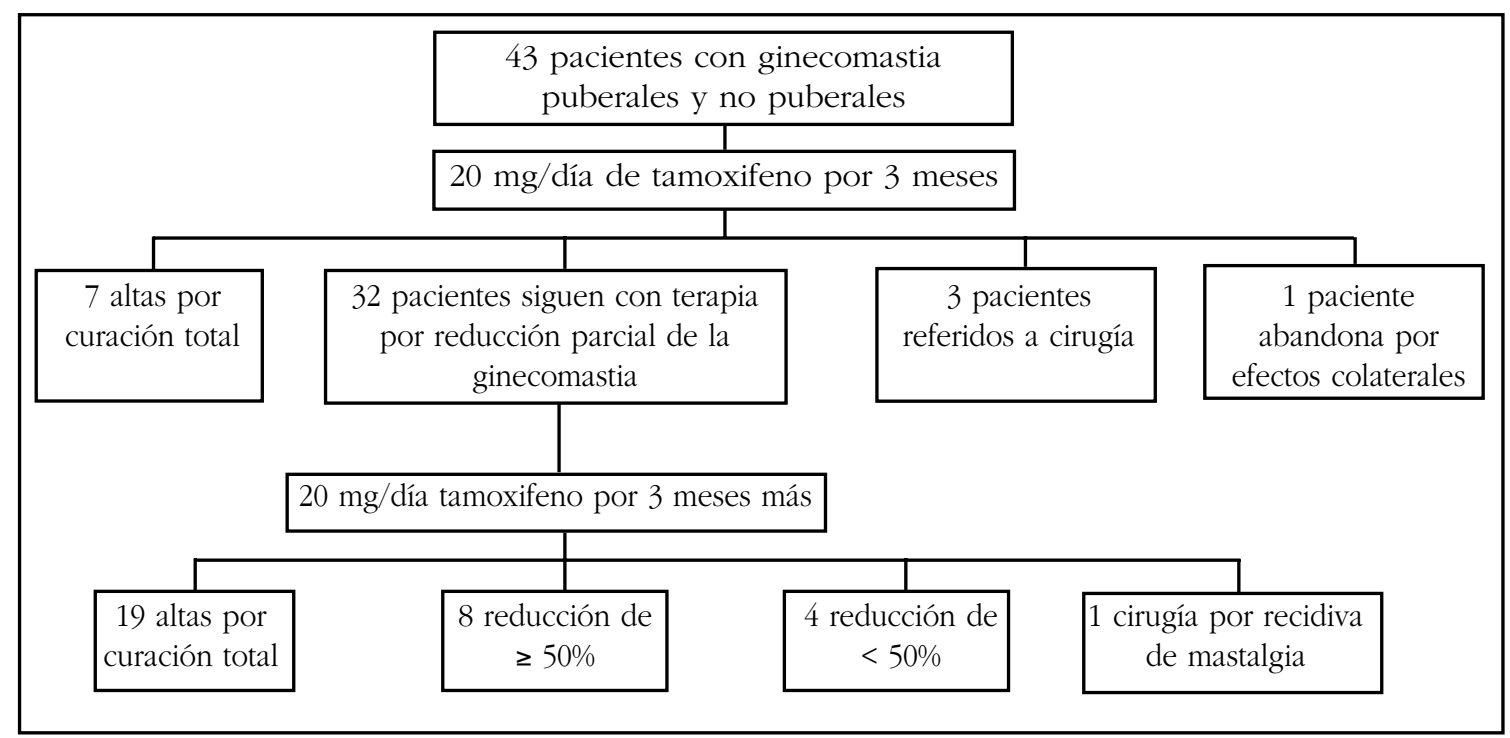

Figura 1. Evolución del tratamiento con tamoxifeno en 43 pacientes con ginecomastia

Al tercer mes, en 16\% (7/43) desapareció la Gi, en 49\% (21/43) la reducción del tamaño fue $\geq 50 \%$ y en $35 \%(15 / 43)$ la reducción de tamaño fue $<50 \%$. Se enviaron a cirugía 3 pacientes por el impacto psicológico que provocaba su Gi no reducida por la terapia; 1 paciente abandonó por cuadro diarreico que relacionó con el tamoxifeno.

Los 32 pacientes restantes se trataron por otros 3 meses, completando 6 meses de terapia continua, desapareciendo la Gi en 59\% (19/32) p $=0.001$, el tamaño disminuyó en $\geq 50 \%$ en $25 \%$ de ellos (8/32) y en $<50 \%$ en $13 \%$ (4/32); un paciente fue referido a cirugía por recidiva de mastalgia (Figura 1).

En $62 \%$ del total del grupo (26/42) desapareció la Gi y en 95\% (19/20) el dolor.

Clasificando los resultados de todos los pacientes en bueno, regular y malo, obtuvimos desaparición de dolor y Gi (bueno) en 62\% (26/ 42), reducción de Gi en $\geq 50 \%$ (regular) en 19\% (8/ 42) y reducción $<50 \%$ y referencia a cirugía (mala) en 19\% (8/42).

Analizamos separadamente el resultado de la terapia con tamoxifeno en Gi puberal (Tabla 1) y no puberal (Tabla 2), obteniéndose resultado

Tabla 1. Evolución del resultado de la terapia con tamoxifeno en ginecomastia puberal

\begin{tabular}{|lccccc|}
\hline & $\begin{array}{c}\text { Dolor } \\
\text { No/ total }\end{array}$ & $\%$ & Tamaño & № / total & $\%$ \\
\hline Inicial & $12 / 27$ & 44 & & $27 / 27$ & 100 \\
& & & Desaparece Gi & $4 / 27$ & 15 \\
3 Meses & $0 / 27$ & 0 & $\downarrow$ tamaño $\geq 50 \%$ & $13 / 27$ & 48 \\
& & & $\downarrow$ tamaño $<50 \%$ & $10 / 27$ & 37 \\
& & & Desaparece Gi & $12 / 20$ & 60 \\
6 meses & \multirow{2}{*}{$1 / 20$} & 5 & $\downarrow$ & $5 / 20$ & 25 \\
& & & $\downarrow$ tamaño $\geq 50 \%$ & $3 / 20$ & 15 \\
\hline
\end{tabular}


Tabla 2. Evolución del resultado de la terapia con tamoxifeno en ginecomastia patológica

\begin{tabular}{|lccccc|}
\hline & $\begin{array}{c}\text { Dolor } \\
\text { No/total }\end{array}$ & $\%$ & Tamaño & № / total & $\%$ \\
\hline Inicial & $8 / 16$ & 50 & & & \\
& & & & $3 / 16$ & 19 \\
3 meses & $0 / 16$ & 0 & $\downarrow$ tamaño $\geq 50 \%$ & $7 / 16$ & 44 \\
& & $\downarrow$ tamaño $<50 \%$ & $6 / 16$ & 37 \\
6 meses & & Desaparece Gi & $7 / 12$ & 58 \\
& $0 / 12$ & 0 & $\downarrow$ tamaño $\geq 50 \%$ & $4 / 12$ & 33 \\
\end{tabular}

Tabla 3. Tamaño de la ginecomastia y resultado del tratamiento con tamoxifeno

\begin{tabular}{|c|c|c|c|c|c|c|}
\hline \multicolumn{4}{|c|}{ ㄴ cm diámetro máximo } & \multicolumn{2}{|c|}{ <4 cm diámetro máximo } & $\%$ \\
\hline Inicial & & $33 / 43$ & 77 & & $10 / 43$ & 23 \\
\hline 6 meses & Desaparece Gi & $17 / 33$ & 52 & Desaparece Gi & $9 / 10$ & $90^{*}$ \\
\hline
\end{tabular}

$*(p=<0,05)$.

Tabla 4. D uración de ginecomastia y resultado de tratamiento con tamoxifeno en 39 pacientes

\begin{tabular}{|c|c|c|c|c|}
\hline \multirow[b]{2}{*}{ Respuesta } & \multicolumn{2}{|c|}{$\leq 2$ años de duración } & \multicolumn{2}{|c|}{$>2$ años de duración } \\
\hline & no/total & $\%$ & $\mathrm{n}$ o/total & $\%$ \\
\hline Buena & $21 / 30$ & $70^{*}$ & $5 / 9$ & $56^{*}$ \\
\hline Regular & $5 / 30$ & 17 & $1 / 9$ & 11 \\
\hline Mala & $4 / 30$ & 13 & $3 / 9$ & 33 \\
\hline
\end{tabular}

*NS (Fisher exact test).

bueno en 59\% (16/27) de Gi fisiológica de la pubertad y en 63\% (10/16) de Gi no puberal (patológica e idiopática del adulto) $\mathrm{p}=>0,05$ (NS).

Efectos colaterales en 2/43 (diarreas y bochornos). La Tabla 3 señala que con tamaño inicial de $\geq 4 \mathrm{~cm}$ de diámetro máximo, en 52\% (17/33) desaparece la Gi con 6 meses de terapia; con $<4$ $\mathrm{cm}$ esto ocurre en 90\% ( $9 / 10)(\mathrm{p}=<0,05)$. En la Tabla 4 se relaciona duración de la Gi, con el resultado de la terapia. En los de duración de $\leq 2$ años desapareció la Gi en 70\% (21/30) y en los de $>2$ años 56\% (5/9) p = > 0,05 (NS). Un 27\% de los pacientes (7/26) recidivó dentro del año post desaparición de la Gi.

Los 6 pacientes cuya Gi se produjo por los antiandrógenos indicados por acné severo, continuaron esa terapia más el agregado de tamoxifeno, lográndose su desaparición y la remisión del acné. 


\section{DisCUSIÓN}

El tamoxifeno fue eficaz en la desaparición rápida del dolor, resultado concordante con la literatura 15-8,20-23. Sólo en un paciente recidivó.

La reducción del volumen fue evaluada por palpación y medición mamaria con regla al igual que todos los autores con que nos comparamos en la Tabla 5.

Es posible que la ultrasonografía sea más precisa para evaluar los cambios de tamaño, aunque el autor que la utilizó ${ }^{25}$ señala que este punto no está definido. Los resultados de disminución de volumen concuerdan con la bibliografía ${ }^{15-}$ 17,20-24 y difiere de otro ${ }^{18}$, en este último la escasa reducción de tamaño se debería a un breve período de terapia.

Una limitación de nuestro trabajo y la mayoría de las publicaciones, es no contar con un grupo de control durante la terapia, lo que se explica por el problema ético de no tratar síntomas como dolor, repercusión psicosocial, etc.

Nuestro trabajo se ubica entre los de mayor número de pacientes y aplicó un criterio más estricto de respuesta terapéutica, al clasificar como regular la disminución del 50\% o más del volumen mamario, lo que otros estiman satisfactorio midiendo con regla ${ }^{24}$ o ultrasonografía 25 . Los pa- cientes consideraron satisfactoria una reducción de $>50 \%$ aunque no existiera desaparición total, ya que por ejemplo, pasar de un diámetro máximo de $4 \mathrm{~cm}$ a $1 \mathrm{~cm}$, resulta en un volumen residual mamario poco notorio.

La desaparición completa y la reducción de más del 50\% señalan que la terapia es clínicamente eficaz en más del 80\% de los casos. La recidiva en 27\% de los casos fue algo superior al 23\% de Ting ${ }^{20}$.

Los escasos efectos colaterales concuerdan con lo publicado ${ }^{13-16,19-22}$.

Los autores que analizan el resultado de antiestrógenos o inhibidores de aromatosa en terapia de Gi puberal, discuten la confiabilidad de sus resultados por la remisión espontánea de la Gi puberal en el período \pm 2 años desde su inicio. Esto explicaría el no encontrar diferencias significativas al comparar anastrozole (inhibidor de la aromatasa) versus placebo ${ }^{25}$.

Similar situación podría haber ocurrido en nuestras Gi puberales, ya que por síntomas agudos (mastalgia, crecimiento rápido etc.) iniciamos tratamiento antes de que se cumplieran 2 años de evolución en $2 / 3$ de ellos.

No hubo diferencias en la respuesta terapéutica entre Gi fisiológica puberal y no puberal (patológica e idiopática del adulto), lo que sugiere en ambos casos la existencia de un desbalance

Tabla 5. Resultados de tratamiento con tamoxifeno. Comparación con otras casuísticas

\begin{tabular}{|c|c|c|c|c|c|c|}
\hline & $\begin{array}{l}\text { Tamoxifeno } \\
\text { mg/día }\end{array}$ & $\begin{array}{l}\text { Tratamiento } \\
\text { meses }\end{array}$ & $\begin{array}{c}\mathrm{n}^{\mathrm{o}} \\
\text { Pacientes }\end{array}$ & $\begin{array}{l}\text { Resultado } \\
\text { total }\end{array}$ & $\mathrm{n}^{\mathrm{O}}$ & $\%$ \\
\hline Ting & 20 & $1-12$ & 23 & $\begin{array}{c}\text { Desaparece Gi } \\
\text { Desaparece Dolor }\end{array}$ & $\begin{array}{c}18 / 23 \\
9 / 11\end{array}$ & $\begin{array}{l}78 \\
82\end{array}$ \\
\hline Parker & 10 & 1 & 10 & $\begin{array}{c}\text { Desaparece Gi } \\
\text { Desaparece Dolor }\end{array}$ & $\begin{array}{l}7 / 10 \\
4 / 4\end{array}$ & $\begin{array}{c}70 \\
100\end{array}$ \\
\hline MacDermott & 20 & $2-4$ & 6 & $\begin{array}{c}\text { Desaparece Gi } \\
\text { Desaparece Dolor }\end{array}$ & $\begin{array}{l}3 / 6 \\
5 / 6\end{array}$ & $\begin{array}{l}50 \\
83\end{array}$ \\
\hline Alagaratnam & 40 & $1-4$ & 61 & $\begin{array}{c}\text { Desaparece Gi } \\
\text { Desaparece Dolor }\end{array}$ & $\begin{array}{l}49 / 61 \\
49 / 49\end{array}$ & $\begin{array}{c}80 \\
100\end{array}$ \\
\hline Eversmann & 20 & $2-4$ & 16 & $\begin{array}{c}\text { Desaparece Gi } \\
\text { Desaparece Dolor }\end{array}$ & $\begin{array}{l}14 / 16 \\
10 / 11\end{array}$ & $\begin{array}{l}88 \\
91\end{array}$ \\
\hline Konig & $20-40$ & $2-12$ & 10 & Desaparece Gi & $8 / 10$ & 80 \\
\hline Devoto & 20 & 6 & 42 & $\begin{array}{c}\text { Desaparece Gi } \\
\text { Desaparece Dolor }\end{array}$ & $\begin{array}{l}26 / 42 \\
19 / 20\end{array}$ & $\begin{array}{l}62 \\
95\end{array}$ \\
\hline
\end{tabular}


andrógeno estrógeno en tejido mamario el que lograría ser revertido por el antiestrógeno. El estrógeno en el hombre es sintetizado fundamentalmente en sitios extragonadales como la mama, transformando el andrógeno en estrógeno por la enzima aromatasa ${ }^{26}$. Los inhibidores de aromatasa se utilizan para tratar $\mathrm{Gi}^{25,27}$ obteniéndose resultados menos eficaces que con los antiestróge$\operatorname{nos}^{28,29}$, lo que supondría que el bloqueo del receptor estrogénico es más importante que la disminución del estrógeno plasmático e intramamario.

Fue exitoso el combinar tamoxifeno con el antiandrógeno que produjo Gi durante la terapia del acné. Resultados similares se reportaron con tamoxifeno-flutamida ${ }^{30}$ y tamoxifeno-bicalutami$\mathrm{da}^{29}$ en Gi desarrollada durante terapia con antiandrógenos en cáncer prostático. Se ha señalado que las Gi con diámetro máximo $\geq 4 \mathrm{~cm}$ no regresarían con antiestrógenos ${ }^{18}$, nuestro $52 \%$ de desaparición de $\mathrm{Gi} \geq 4 \mathrm{~cm}$ indicaría que el mayor tamaño no impide utilizarlos. Se recomienda no

\section{REFERENCIAS}

1. Hershrovitz E, Leiberman E. Gynecomastia: A Review. The Endocrinologist 2002; 12: 321-32.

2. Mathur R, Braunstein G. Gynecomastia: Pathomechanisms and treatment strategies. Horm Res 1997; 48: 95-102.

3. Devoto E, Madariaga M, Aravena L, Lioi X. Etiología de la ginecomastia. Importancia de no subdiagnosticar una ginecomastia patológica. Rev Méd Chile 2007; 135: 189-97.

4. Gabrilove J, Sharma D, Wotiz H, Dorfman R. Feminizing adrenocortical tumors in the male, a review of 52 cases including a case report. Medicine 1965; 44: 37-79.

5. Mineur P, De Cooman S, Hustin J, Verhoeven G, De Hertogh R. Feminizing Testicular Leydig Cell Tumor: Hormonal profile before and after unilateral orchidectomy. J Clin Endocrinol Metab 1987; 64: 686-91.

6. Smyth C, Bremner W. Klinefelter Syndrome. Arch Intern Med 1998; 158: 1309-14.

7. Nydick M, Bustos J, Dale J, Rawson R. Gynecomastia in adolescent boys. JAMA 196; 178: 449-54. tratar médicamente la Gi de más de 2 años de duración, ya que a mayor duración existirían lesiones morfológicas que difícilmente regresarían $2,26,31,32$

Diferimos de esa afirmación, ya que en $56 \%$ de Gi de $>2$ años se logró buen resultado, lo que justifica realizar la terapia. El tamoxifeno utilizado por 6 meses en forma continua, se demostró eficaz en la desaparición del dolor y de la Gi en un porcentaje importante de los pacientes, con escasos síntomas colaterales, constituyéndose en una alternativa a la cirugía.

La combinación del fármaco causante de Gi con tamoxifeno, permitió su desaparición.

Ni el mayor tamaño de la Gi, ni su duración, contraindicarían realizar la terapia antiestrogénica.

\section{Agradecimientos}

Los autores agradecen al Dr. Ariel Fuentes (Medicina Reproductiva, IDIMI, U de Chile) por su colaboración en el análisis estadístico.

8. Colombo-Benkmann M, Buse B, Stern J, Herfarth C. Indications for and results of surgical therapy for male Gynecomastia. Am J Surg 1999; 178: 60-3.

9. Macgregor J, Jordan C. Basic guide to the mechanisms of Antiestrogen action. Pharmacological Reviews 1998; 50: 151-96.

10. Davies C, Arriagada R, Ziegler A, Peralta O. Duración del tratamiento adyuvante con Tamoxifeno en el cáncer de mama operable. Rev Méd Chile 1998; 126: 210-7.

11. Stepanas A, Burnet R, Harding P, Wise P. Clomiphene in the treatment of pubertal-adolescent Gynecomastia: A preliminary report. J Pediatr 1977; 90: 651-3.

12. Le Roit D, Sobel R, Glick S. The effect of clomiphene citrate on pubertal Gynaecomastia. Acta Endocrinol 1980; 95: 177-80.

13. Plourde P, Kulin H, Santner S. Clomiphene in the treatment of adolescent Gynecomastia. Am J Dis Child 1983; 137: 1080-2.

14. Devoto E, Aravena L, Madariaga M. Utilidad de la terapia antirreceptor de hormonas sexuales en la mujer y en el varón. Rev SOGIA 1995; 2: 16-22. 
15. JefFerys DB. Painful Gynaecomastia treated with Tamoxifen. Br Med J 1979; 1: 1119-20.

16. Parker L, Gray D, Lai M, Levin E. Treatment of Gynecomastia with Tamoxifen: A double-blind crossover study. Metabolism 1986; 35: 705-8.

17. Serels S and Melman A. Tamoxifen as treatment for Gynecomastia and mastodynia resulting from hormonal deprivation. J Urol 1998; 159: 1309.

18. McDermott MT, Hofeldt FD, Kidd GS. Tamoxifen therapy for painful idiopathic Gynecomastia. South Med J 1990; 83: 1283-5.

19. Niewoehner C, Nuttal F. gynecomastia in a hospitalized male population. Am J Med 1984; 77: 633-8.

20. Ting A, Chow L, Leung Y. Comparison of Tamoxifen with Danazol in the management of idiopathic Gynecomastia. Am Surg 2000; 66: 38-40.

21. Alagaratnam TT. Idiopathic Gynecomastia treated with Tamoxifen: A preliminary report. Clin Ther 1987; 9: 483-7.

22. Konig R, Schonberger W, Neumann P, Benes P, Grimm W. Treatment of marked Gynecomastia in puberty with Tamoxifen. Klin Padiatr 1987; 199: 389-91.

23. Khan Hn, Rampaul R, Blamey Rw. Management of physiological gynaecomastia with tamoxifen. The Breast 2004; 13: 61-5.

24. Lawrence S, Faught A, Vethamuthu J, Lawson M. Benefical Effects of Raloxifene and Tamoxifen in the Treatment of Pubertal Gynecomastia. J Pediatr 2004; 145: 71-6.

25. Plourde P-V, Reiter EO, Hann-Chang JOU, Desrochers P, Rubin S, Bercu B et al. Safety an Efficacy of Anastrozole for the Treatment of Pubertal Gynecomastia: A Randomized, Double-Blind, Placebo-Controlled Trial. J Clin Endocrinol Metab 2004; 89: 4428-33.

26. Simpson E, Misso M, Hewitt K, Hill R, Boon W, Jones $\mathrm{M}$ ET Al. Estrogen - the Good, the Bad, and the unexpected. Endocrine Reviews 2005; 26: 322-30.

27. Riepe F, Baus I, Wiest S, Krone N, Sippell W, Partsch C-J. Treatment of Pubertal Gynecomastia with the Specific aromatase Inhibitor Anastrozole. Horm Res 2004; 62: 113-8.

28. Boccardo F, Rubagotti A, Battaglia M, Di Tonno P, Selvaggi F, Conti G ET al. Evaluation of Tamoxifen and Anastrazole in the Prevention of Gynecomastia and Breast Pain Induced by Bicalutamide Monotherapy of Prostate Cancer. J Clin Oncology 2005; 23: 808-15.

29. Saltzstein D, Sieber P, Morris T, Gallo J. Prevention and management of bicalutamide - induced gynecomastia and breast pain: randomized endocrinologic and clinical studies with tamoxifen and anastrazole. Prostate Cancer and Prostatic Diseases 2005; 8: 75-83.

30. Staiman V, Lowe F. Tamoxifen for Flutamide/ Finasteride induced Gynecomastia. Urol 1997; 50: 929-33.

31. Braunstein G. Gynecomastia. N Engl J Med 1993; 328: 490-5.

32. Nicolis G, Modlinger R, Gabrilove JL. A study of the histopathology of human Gynecomastia. $J$ Clin Endocrinol Metab 1971; 32: 173-8. 